Journal of Advanced College of Engineering and Management, Vol. 4, 2018

\title{
BALANCING NUMBERS AND APPLICATION
}

\author{
Ramesh Gautam \\ Lecturer of Mathematics, RR Campus, Tribhuvan University, Kathmandu, Nepal \\ E-mail Address: rgnumberth79@gmail.com
}

\begin{abstract}
In this paper, we present about origin of Balancing numbers; It's connection with Triangular, Pells numbers, and Fibonacci numbers; beginning with connections of balancing numbers with other numbers system, It elaborate the different generating functions of balancing numbers. It also include some amazing recurrence relations; and the application of balancing numbers in solving Diophantine equation.
\end{abstract}

Keywords: Triangular numbers, Pells numbers, Balancing numbers, Balancers, Fibonacci numbers

\section{Introduction}

Balancing numbers is a natural number ' $\mathrm{N}$ ' which satisfies the simple Diophantine equation: $1+2+$ $3+\cdots+\mathrm{N}-1=(\mathrm{N}+1)+(\mathrm{N}+2)+\cdots+(\mathrm{N}+\mathrm{r})$, where $\mathrm{r}$ is called balancer; for example, $\mathrm{N}=6,35,204$ are balancing numbers and $\mathrm{r}=2,14$, and 84 respectively are balancers; A. Behera and G.K Panda [1] introduced the concept of balancing numbers in 1998.

The concept of balancing numbers is closely related with the established triangular numbers; the positive integer $\mathrm{N}$ is called balancing number if and only if $N^{2}$ is a triangular number; the only triangular numbers whose square is also triangular are 1 and 6 ; similarly a number $\mathrm{N}$ is called balancing if and only if $8 N^{2}+1$ is perfect square.

Even if from the definition of balancing numbers, balancing numbers are positive integers bigger than 1,1 is accepting as balancing number because $8.1 .1+1=9$ is perfect square.A. Behera and G.K Panda [1] proved that some balancing numbers are even and some others are odd; just like sum of first odd ' $\mathrm{N}$ ' natural numbers is $N^{2}$, Panda showed that the sum of first odd $\mathrm{N}$ balancing numbers is equal to the square of the Nth balancing number; this property is not true for Fibonacci numbers; there no sequence balancing number in the Fibonacci sequence.

The search for balancing numbers is well known integer sequence was first initiated by Liptai[11]; he proved that there is no balancing number in the Fibonacci sequence other than 1; he further showed that sequence balancing numbers in odd natural numbers are the sums of two consecutive balancing numbers.

Panda[13] generalized balancing numbers by introducing sequence balancing numbers, in which, the sequence of numbers, used in the definition of balancing numbers is replaced by an arbitrary sequence of real numbers; thus if $\left\{a_{n}\right\}$ be a sequence real numbers, then $a_{n}$ is called a sequence of balancing number if $a_{1}+a_{2}+a_{3}+\cdots_{. .}+a_{n-1}=a_{n+1}+a_{n+2}+\cdots_{. .}+a_{n+r}$ for some natural number $\mathrm{r}$.

Panda also introduced the concept of higher order balancing number ; Panda[13] call the higher order balancing numbers, the balancing squares and balancing cubes; in As shown by Behera and Panda[1], the square of a balancing number is a triangular number, and indeed, all square triangular numbers can be generated in this way. 
In mathematics a continued fraction is an expression obtained through an iterative process of representing a number as the sum of its integer part and the reciprocal of another number, then writing this other number as the sum of its integer part and another reciprocal, and so on. For any real number $\alpha$, we can write $\alpha=a_{0}+A_{0}$, where $a_{0} \in Z$ is lower integral part of is $\alpha$ and $A_{0}$ is the fractional part of $\alpha$. If $A_{0}$ is zero then, the algorithm of continued fraction is stop. Otherwise, $0<A_{0}<1$, then we continue with $\frac{1}{A_{0}}=\alpha_{1}>1$. Again writing $\alpha_{1}=a_{1}+A_{1}$, where $a_{1}$ is the lower integral part of $\alpha_{1}$ and $A_{1}$ is the fractional part of $\alpha_{1}$. If $A_{1}=0$, then the algorithm stop. Otherwise we can proceed and obtain the continued fraction expansion of $\alpha$.

$$
\begin{aligned}
& \alpha=a_{0}+\frac{1}{\alpha_{1}} \\
& \alpha=a_{0}+\frac{1}{a_{1}+\frac{1}{a_{2} \frac{1}{\ddots+a_{n-1}+\frac{1}{\alpha_{n}}}}}
\end{aligned}
$$

For, $n>0, a_{n} \in Z+$. The continued fraction expansion of real number terminate after finite iterations if and only if it is rational. Whenever it goes infinitely many iterations if and only if it is irrational number.

An infinite sequence $a_{0}, a_{1}, a_{2} \ldots$ of integers, all positive except perhaps for $a_{0}$ determines an infinite simple continued fraction. The value of $\left[a_{0}, a_{1}, a_{2}, \ldots\right]$ is defined to be

$$
\lim _{n \rightarrow \infty}\left[a_{0}, a_{1}, \ldots, a_{n}\right]=\lim _{n \rightarrow \infty} \frac{P_{n}}{Q_{n}}
$$

\subsection{Convergent, Partial Quotients and Equivalent Irrational Numbers}

The rational number, defined by $\frac{P_{n}}{Q_{n}}=\frac{a_{n} P_{n-1}+P_{n-2}}{a_{n} Q_{n-1}+Q_{n-2}}$,

is called the $\mathrm{n}_{\mathrm{th}}$ convergent of $\alpha$, where $P_{-2}=0, P_{-1}=1, Q_{-2}=1, Q_{-1}=0$ gives $P_{0}=a_{0}, Q_{0}=1$

The integer $a_{n}$ in the continued fraction expansion is called $\mathrm{n}_{\text {th }}$ partial quotient and $\alpha_{n}=$ $\left[a_{n}, a_{n+1}, a_{n+2}, \ldots\right]$ is called the $\mathrm{n}_{\mathrm{th}}$ total quotient [4].

2.3. Recurrence Relation [3, 7, 12]: In mathematics, a recurrence relation is an equation that defines a sequence recursively; each term of the sequence is defined as a function of the preceding terms.

2.4. Fibonacci Sequence $[4,6,8,17,18]$ :The first two Fibonacci numbers are $F_{1}=1, F_{2}=$ $1, F_{n+1}=F_{n}+F_{n-1}, n \geq 2$ for other terms of the sequence.

2.5. Pell Sequence [5]: The first two Pell numbers $P_{1}=1, P_{2}=2$ are and other terms of the sequence are obtained by means of the recurrence relation; In fact these are the numbers involving in the numerator of theconvergent of the continued fractions of quadratic irrationals. 
2.5. Pell Sequence [5]: The first two Pell numbers $P_{1}=1, P_{2}=2$ are and other terms of the sequence are obtained by means of the recurrence relation; In fact these are the numbers involving in the numerator of theconvergent of the continued fractions of quadratic irrationals.

$$
P_{n+1}=2 P_{n}+P_{n-1}, n \geq 2
$$

2.6. Associated Pell Sequence [5]: Associated Pell sequence is also obtained from the same recurrence relation as that of Pell numbers. The first two associated Pell numbers are and other terms of the sequence are obtained by $Q_{1}=1, Q_{2}=3$

means of the recurrence relation; In fact these are the numbers involving in the denominators of the convergent continued fractions of quadratic irrationals.

$$
Q_{n+1}=2 Q_{n}+Q_{n-1}, n \geq 2
$$

2.7. Triangular Number: A positive integer is called triangular numbers if it can be obtained from $\frac{N(N+1)}{2} ; \mathrm{N}$ is positive integer. The numbers $1,3,6,10 \ldots$ are triangular numbers. The justifications for the name triangular number are many. One such reason

may be the fact that the triangular number $\frac{N(N+1)}{2}$ represents the area of aright angled triangle with base $(N+1)$ and perpendicular $N$. It is well known that a positive integer $\mathrm{N}$ is a triangular number if and only if $8 \mathrm{~N}+1$ is a perfect square.

2.8. Square Triangular Number: A number which is triangular as well as a square is called a square triangular number [2, 19]. Examples of square triangular numbers are 1, 36, 1225 and so on. While the triangular numbers satisfy the recurrence relation $T_{1}=1, T_{n+1}=T_{n}+n+1, n \geq 2$ and the square triangular numbers satisfy the recurrence relation:

$S_{1}=1, S_{2}=36$ and $S_{n+1}=34 S_{n}-S_{n-1}+2, n \geq 2[14,15]$.

$S_{n+1}=34 S_{n}-S_{n-1}+2, n \geq 2$.

2.9. Pell's Equation [9, 10, 16]: The equation $x^{2}-d y^{2} \pm 1$ to be solved in

positive integers $\mathrm{x}$ and $\mathrm{y}$ for a given positive integer $\mathrm{d}$ (which is not a perfect square), is called the Pell's equation. For example, for $\mathrm{d}=5$ one can take $\mathrm{x}=9, \mathrm{y}=4$.

\section{Some Properties of Balancing Numbers [1]}

Property 3.1. A positive integer $\mathrm{N}$ is a balancing number if and only if $N^{2}$ is triangular number.

Proof: Let $\mathrm{N}$ is a balancing number then using definition: $1+2+3+\ldots+\mathrm{N}-1=(\mathrm{N}+1)+(\mathrm{N}+2)+\ldots+$ $(\mathrm{N}+\mathrm{r})$.

$\Leftrightarrow 1+2+3+\ldots+\mathrm{N}-1=1+2+3+\ldots+\mathrm{N}-1+\mathrm{N}+(\mathrm{N}+1)+(\mathrm{N}+2)+\ldots+(\mathrm{N}+\mathrm{r})-(1+2+3+\ldots+\mathrm{N}-1+\mathrm{N})$

$\Leftrightarrow \frac{N(N-1)}{2}=\frac{(N+r)(N+r+1)}{2}-\frac{N(N+1)}{2} \Leftrightarrow N^{2}=\frac{(N+r)(N+r+1)}{2}$.

Which shows that $N^{2}$ is a triangular number.

Property 3.2. The positive integer $\mathrm{N}$ is a balancing number if and only if $8 N^{2}+1$ is a perfect square.

Proof: If $\mathrm{N}$ is a balancing number then by using (1)

$$
\begin{array}{r}
N^{2}=\frac{(N+r)(N+r+1)}{2} \\
r^{2}+(2 N+1) r-\left(N^{2}+N\right)=0 \ldots
\end{array}
$$


Equation (2) is quadratic in $\mathrm{r}$, then $r=\frac{-(2 N+1)+\sqrt{1+8 N^{2}}}{2}$

From (3) it is clear that $8 N^{2}+1$ must be perfect square for $r$ being balancer is always positive integer.

\section{Functions Generating Balancing Numbers [1]}

For any balancing number $\mathrm{x}, \mathrm{F}(\mathrm{x}), \mathrm{G}(\mathrm{x})$ and $\mathrm{H}(\mathrm{x})$ defined by

(a) $\quad F(x)=2 x \sqrt{1+8 x^{2}}$ is even balancing number

(b) $\quad G(x)=3 x+\sqrt{1+8 x^{2}}$ is even and odd depending on $\mathrm{x}$ odd and even.

(c) $\quad H(x)=17 x+6 \sqrt{1+8 x^{2}}$ is even and odd according as $\mathrm{x}$ even and odd.

(d) $\quad K(x)=6 x \sqrt{1+8 x^{2}}+16 x^{2}+1$ is an odd balancing number.

Are also balancing numbers.

Proof (a): Since $\mathrm{x}$ is balancing number, $1+8 x^{2}$ is perfect square and $\frac{8 x^{2}}{2}\left(8 x^{2}+1\right)=4 x^{2}\left(8 x^{2}+1\right)$ is a triangular number which is also a perfect square; therefore it is a square root $2 x \sqrt{1+8 x^{2}}$ is balancing number, which is even. Thus, for any given balancing number $\mathrm{x}, 2 x \sqrt{1+8 x^{2}}$ is even balancing number.

(b) Since $1+8 x^{2}$ is a perfect square, it follows that

$$
\begin{gathered}
8[G(x)]^{2}+1=8\left[3 x+\sqrt{1+8 x^{2}}\right]^{2}+1=8\left[9 x^{2}+6 x \sqrt{1+8 x^{2}}+8 x^{2}+1\right]+1 \\
=72 x^{2}+48 x \sqrt{1+8 x^{2}}+64 x^{2}+9 \\
=64 x^{2}+48 x \sqrt{1+8 x^{2}}+9\left(1+8 x^{2}\right) \\
=\left(8 x+3 \sqrt{1+8 x^{2}}\right)^{2}
\end{gathered}
$$

Thus, $8[G(x)]^{2}+1$ is perfect square; which claims that $\mathrm{G}(\mathrm{x})$ is also balancing number.

(c)Again, $\mathrm{G}(\mathrm{G}(\mathrm{x}))=\mathrm{G}\left(3 x+\sqrt{1+8 x^{2}}\right)[$

$$
\begin{aligned}
& =3\left[3 x+\sqrt{1+8 x^{2}}\right]+\sqrt{8\left(3 x+\sqrt{1+8 x^{2}}\right)^{2}+1} \\
& =9 x+3 \sqrt{1+8 x^{2}}+\sqrt{8\left(9 x^{2}+6 x \sqrt{1+8 x^{2}}+8 x^{2}+1\right)+1} \\
& =9 x+3 \sqrt{1+8 x^{2}}+\sqrt{72 x^{2}+48 x \sqrt{1+8 x^{2}}+64 x^{2}+8+1} \\
& =9 x+3 \sqrt{1+8 x^{2}}+\sqrt{\left(8 x+3 \sqrt{1+8 x^{2}}\right)^{2}} \\
& =9 x+3 \sqrt{1+8 x^{2}}+8 x+3 \sqrt{1+8 x^{2}} \\
& =17 x+6 \sqrt{1+8 x^{2}} \\
& =H(x)
\end{aligned}
$$

Thus $\mathrm{H}(\mathrm{x})$ is also balancing number

(d) $\quad \mathrm{K}(\mathrm{x})=\mathrm{G}(\mathrm{F}(\mathrm{x}))=6 x \sqrt{1+8 x^{2}}+16 x^{2}+1$, proof is exactly similar with proof of $(\mathrm{C}$ 
Remark: 1It is important to note that, if $\mathrm{x}$ is any balancing number, then $\mathrm{F}(\mathrm{x})$ is always even, whereas $G(x)$ is even when $x$ is odd and $G(x)$ is odd when $x$ is even. $H(x)$ is even for $x$ even and odd for $x$ odd and $\mathrm{K}(\mathrm{x})$ is always odd balancing number.

2 If $\mathrm{x}$ is any balancing number, then the next balancing number is $3 x+\sqrt{1+8 x^{2}}$ and the previous one is $3 x-\sqrt{1+8 x^{2}}$.

\section{Recurrence Relations of Balancing Numbers}

$\mathrm{X}$ is a balancing number if and only if $1+8 x^{2}$ is a perfect square; there exist an positive integer $\mathrm{y}$ such that $1+8 x^{2}=y^{2} \Rightarrow y^{2}-8 x^{2}=1$

Now our interest to find a balancing number is reduced to solve the Pell's equation for $\mathrm{d}=8$ and by using the continued fraction expansion of $\sqrt{8}$, we can obtain the fundamental solution $x_{1}=1, y_{1}=3$; all other infinite solutions can be obtained by using the formula:

$y_{n}+\sqrt{8} x_{n}=\left[3+\sqrt{8}^{n} \ldots \ldots \ldots \ldots(5)\right.$, where ' $n$ ' is any non-zero integer;

from (5) we can obtain, $y_{n}-\sqrt{8} x_{n}=\left[3-\sqrt{8}^{n}\right.$

Now subtracting (6) from (5) we have the nth balancing number,

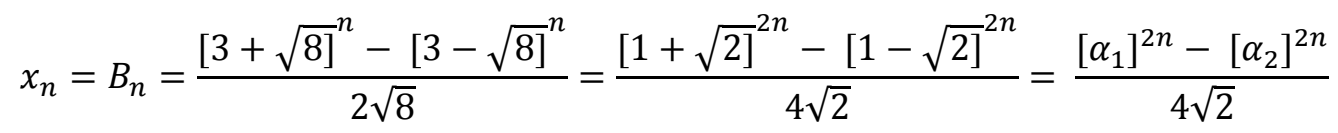

where, $\alpha_{1}=1+\sqrt{2}$ and $\alpha_{2}=1-\sqrt{2}$.

Theorem: If $B_{n}$ is nth balancing number, then

(1) $B_{n+1}=6 B_{n}-B_{n-1}, n \geq 2$

(2) $B_{n}{ }^{2}=1+B_{n-1} B_{n+1}, n \geq 2$

(3) Any three consecutive balancing numbers are approximately in geometric progression.

(4) For two positive integer $\mathrm{m}$ an $\mathrm{n}, B_{m+n}=B_{m} B_{n+1}-B_{m-1} B_{n}$

(5) $B_{2 n-1}=B_{n}{ }^{2}-B_{n-1}{ }^{2}$

(6) $\quad B_{2 n}=B_{n}\left[B_{n+1}-B_{n-1}\right]$

(7) If $\mathrm{n}$ is a natural number then: $B_{1}+B_{3}+\cdots+B_{2 n-1}=B_{n}{ }^{2}$

(8) For $\mathrm{n}$ is a natural number: $B_{2}+B_{4}+\cdots+B_{2 n}=B_{n} B_{n+1}$

Proof: $B_{n+1}+B_{n-1}=\frac{\left[\alpha_{1}\right]^{2(n+1)}-\left[\alpha_{2}\right]^{2(n+1)}}{4 \sqrt{2}}+\frac{\left[\alpha_{1}\right]^{2(n-1)}-\left[\alpha_{2}\right]^{2(n-1)}}{4 \sqrt{2}}$

$$
\begin{aligned}
& =\frac{\left[\alpha_{1}\right]^{2 n}\left[\alpha_{1}{ }^{2}+\alpha_{2}{ }^{2}\right]-\left[\alpha_{2}\right]^{2 n}\left[\alpha_{1}{ }^{2}+\alpha_{2}{ }^{2}\right]}{4 \sqrt{2}} \\
& =\frac{6\left[\alpha_{1}\right]^{2 n}-\left[\alpha_{2}\right]^{2 n}}{4 \sqrt{2}} \\
& =6 B_{n}(\mathrm{n} \geq 2)
\end{aligned}
$$

Alternatively: If $B_{n}$ is a balancing number then by using the results of generating functions:

$$
B_{n+1}=3 B_{n}+\sqrt{1+8 B_{n}^{2}}
$$

And

$$
B_{n-1}=3 B_{n}-\sqrt{1+8 B_{n}^{2}}
$$


Adding these two results gives: $B_{n+1}+B_{n-1}=6 B_{n}$

\section{An Application of Balancing Numbers to Solve Diophantine Equations}

Let us consider the particular type of Diophantine equation: $x^{2}+(x+1)^{2}=y^{2}$ and our interest is to find the solutions in terms of balancing numbers.

Here

$$
\begin{gathered}
x^{2}+(x+1)^{2}=y^{2} \\
2 x^{2}+2(x+1)^{2}=2 y^{2} \\
2 y^{2}-1=(2 x+1)^{2} \\
y^{2}\left(2 y^{2}-1\right)=\frac{2 y^{2}\left(2 y^{2}-1\right)}{2}=y^{2}(2 x+1)^{2}
\end{gathered}
$$

Since both $y^{2}$ and $\left(2 y^{2}-1\right)$ are odd so $y^{2}\left(2 y^{2}-1\right)$ is odd square triangular number and hence $y \sqrt{2 y^{2}-1}$ an odd balancing number say $B=y \sqrt{2 y^{2}-1}$ then $y^{2}\left(2 y^{2}-1\right)=B^{2}$

Then, $2 y^{4}-y^{2}-B^{2}=0$ which gives rises $y^{2}=\frac{1+\sqrt{1+8 B^{2}}}{4}$ so that $y=\frac{\sqrt{1+\sqrt{1+8 B^{2}}}}{2}$

And hence $x=\frac{-1+\sqrt{\left.\frac{1}{2}\left(\sqrt{\left(1+8 B^{2}\right.}\right)-1\right)}}{2}$

Thus in this way balancing numbers are useful to solve the Diophantine equation.

\section{Discussions}

Balancing numbers are connected with triangular numbers, Pells numbers and Fibonacci numbers searching the balancing numbers by using direct definition is tedious; we discussed the generating functions and recurrence relations which play precious role in search more balancing numbers from the given balancing numbers; solving the Diophantine equation is not easy; this paper discussed the application of the balancing numbers to find the solution of some specific Diophantine equation.

\section{Recommendations}

There is another number system like balancing number which is called cobalancing numbers; in the future we can explore the connections of cobalancing number with Fibonacci numbers and associated Pells numbers and other number system; there are various types of generating functions of cobalancing numbers and beautiful recurrence relations. 


\section{References}

1. Behera A. and Panda G. K., "On the Square Roots of Triangular Numbers", The Fibonacci Quarterly 37.2(1999):98-105.

2. Allen B.M., “Squares as Triangular Numbers”, Scripta Math. 20, (1954), 213-214.

3. Balakrishnan V. K., "Introductory Discrete Mathematics”, Courier Dover Publications, 1996.

4. Ball K. M., "Strange Curves, Counting Rabbits, and Other Mathematical Explorations", Princeton University Press, 2003.[6] E. J. Barbeau, Pell's Equation, Springer, 2003.

5. Flannery D., "The square root of 2: A dialogue concerning a number and a sequence", Springer, 2006.

6. Garland T. H., "Fascinating Fibonaccis: Mystery and magic in numbers", Dale Seymour Publications, 1987.

7. Gries D. and Schneider F. B., “A logical approach to discrete math”, Springer,1993.

8. Koshy T., "Fibonacci and Lucas numbers with applications”, Wiley, 2001.

9. Lenstra Jr. H. W., "Solving the Pell equation”, Notices Amer. Math. Soc. 49, (2002) 182-192.

10. $\quad$ Li K. Y., “Pell’s Equation”, Mathematical Excalibur, 6(2001), 1 - 4.

11. Liptai K., “Fibonacci balancing numbers”, Fib. Quart, 42(4) (2004), 330 -340.

12. Nanda S., "Number theory", Allied Publishers, 1985.

13. Panda G. K., "Sequence balancing and cobalancing numbers", The FibonacciQuarterly, 45(2007), $265-271$.

14. Pietenpol J. L, "Square Triangular Numbers”, Amer. Math. Monthly 169,(1962) 168 - 169.

15. Potter D. C. D., “Triangular square number”, Math. Gaz., 56 (1972), 109-110.

16. Tattersall J. J., "Elementary number theory in nine chapters", Cambridge University Press, 1999.

17. Vajda S., "Fibonacci and Lucas numbers, and the golden section: Theory and applications", Dover Publications, 2007.

18. Vorob'ev N. N. and Martin M., “Fibonacci Numbers”, Springer, 2002.

19. Walker G. W., “Triangular Squares”, Problem E 954, Amer. Math. Monthly, 58(1951) 568. 